\title{
Postmenopausal Bleeding: An Update
}

\author{
Shaikh Zinnat Ara Nasreen, Nusrat Mahjabeen, and Safinaz Shahreen
}

\section{ABSTRACT}

The clinical approach to postmenopausal bleeding requires prompt and efficient evaluation to exclude or diagnose endometrial carcinoma and endometrial intraepithelial neoplasia and to find out the real source. Postmenopausal bleeding is 'endometrial cancer until proven otherwise', although only $1-14 \%$ of such patients will actually have cancer. Clinical risk factors of endometrial carcinoma such as obesity, unopposed estrogen use, polycystic ovary syndrome, diabetes mellitus and family history of gynaecologic malignancy also should be considered during evaluation. Postmenopausal bleeding usually attributed to an intrauterine source, but it may arise from the cervix, vagina, vulva or fallopian tubes \& ovaries. The origin of bleeding can also involve non-gynaecologic sites, such as the urethra, bladder, anus/rectum/bowel, or perineum.

Meticulous history and thorough physical examination are must. Initial evaluation is by TVS, if endometrial thickness (ET) is $\langle 4 \mathrm{~mm}$ no further evaluation is required but follow up consultation must. If ET is $>4 \mathrm{~mm}$, hysteroscopic evaluation and endometrial sampling is recommended

Blind endometrial sampling is not accurate as only reveals when endometrial cancer exceeds more than $50 \%$ of the endometrial surface area so may be done if hysteroscopic evaluation is not possible. Higher dose of progesterone may be required for endometrial protection when higher doses of estradiol as hormone replacement therapy are used, or in women with high BMI. Unopposed estrogen therapy is associated with a duration and dose-related increase in risk of endometrial hyperplasia and cancer. Endometrial protection requires an adequate dose and duration of progestogen. Endometrial hyperplasia with atypia has much malignant potential but endometrial hyperplasia without atypia may be managed medically with 3 monthly endometrial sampling, if no regression or further progression hysterectomy is the choice of treatment. Finally, patient counseling with discussion of risks /benefits of different options of treatment modalities is the cornerstone of success of addressing postmenopausal bleeding.

Keywords: Endometrial thickness, endometrial hyperplasia, endometrial cancer, postmenopausal bleeding.

\section{INTRODUCTION}

Postmenopausal bleeding (PMB) is considered at least 12 months after the last normal period. The incidence of postmenopausal bleeding appears to correlate with the time since menopause. Unscheduled bleeding is defined as noncyclical bleeding in menopause women taking hormone replacement therapy. Although PMB usually has benign causes, the priority is to exclude malignancy.

Postmenopausal bleeding is a common clinical problem affecting approximately 5 percent of office gynaecology visits [1].

All postmenopausal women with unexpected vaginal bleeding should be evaluated for endometrial carcinoma since this potentially lethal disease will be the cause of bleeding in approximately 10 percent range (1 to 25 percent), depending upon risk factors [2]. For PMB Endometrial carcinoma must be regarded until proven otherwise. Management of PMB is aimed at excluding
Published Online: January 23, 2021

ISSN: $2593-8339$

DOI: $10.24018 /$ ejmed.2021.3.1.652

\section{Shaikh Zinnat Ara Nasreen*}

Professor \& Head of the Department of Obs. \& Gyne, Z. H. Sikder Womens' Medical College \& Hospital, West Dhanmondi, Dhaka, Bangladesh.

(e-mail: zinnatn@yahoo.com)

Nusrat Mahjabeen

Assistant Professor, Department of Obs. \& Gyne, Z. H. Sikder Womens' Medical College \& Hospital, West Dhanmondi, Dhaka, Bangladesh (e-mail: nusrat.bonny@gmail.com) Safinaz Shahreen

Registrar, Department of Obs. \& Gyne, Luton Hospital, UK.

(e-mail: safinaz@yahoo.com)

*Corresponding Author cervical carcinoma, endometrial carcinoma precancerous endometrial changes and then to treat the real cause. A negative test guarantees the normality; but the clinician should be aware of the possibility of a false negative result. Postmenopausal bleeding usually attributed to an intrauterine source, but may arise from the cervix, vagina, vulva, fallopian tubes, or be related to ovarian pathology (hormone producing tumour).

The origin of bleeding can also involve non-gynaecologic sites, such as the urethra, bladder, anus/rectum/bowel, or perineum. Cervical stenosis may inhibit egress of blood from the uterine cavity; hematometra may result.

The differential diagnosis of bleeding in postmenopausal women is less broad than that for abnormal uterine bleeding in premenopausal women since the various causes of anovulation are not relevant here. 


\section{DifFEREnTial Diagnosis OF PMB}

- Vaginal atrophy. The most common cause of PMB.

- Indiscriminate use of MHT (Menopause hormone therapy), Tamoxifen.

- Endometrial hyperplasia with atypia and without atypia.

- Endometrial cancer.

- Endometrial polyps or Infection.

- Cervical cancer; need to check if the cervical smear is up to date. Cervical polyp.

- Cervical ulcer caused by uterine prolapse.

- Uterine sarcoma (rare).

- Ovarian cancer, especially oestrogen-secreting (theca cell) ovarian tumours.

- Vaginal cancer (very uncommon).

- Vulval cancer may bleed, but the lesion should be obvious.

- Non-gynaecological causes including trauma or a bleeding disorder.

Other causes - lesions and cracks of the vulva, trauma, phytoestrogens, and forgotten pessaries.

Though benign conditions are the most common causes of PMB, but it needs to be considered seriously by the patients and clinicians, as it may be the first warning symptoms of an underlying malignancy, which if diagnosed early can be cured completely.

\section{A. Association of Postmenopausal Bleeding}

Atrophy - Hypoestrogenism causes atrophy of the endometrium and vagina. In the uterus, the collapsed, atrophic endometrial surfaces contain little or no fluid to prevent intra-cavitary friction [3]. This results in micro erosions of the surface epithelium and a subsequent chronic inflammatory reaction (chronic endometritis), which is prone to light bleeding or spotting.

Classic vaginal findings of atrophy include a pale, dry vaginal epithelium that is smooth and shiny with loss of most rugae. If inflammation is present, additional findings may include patchy erythema, petechiae, blood vessels visible through the thinned-out epithelium, friability, bleeding, and discharge.

Cancer - Approximately 6 to 19 percent of women with postmenopausal vaginal bleeding have endometrial cancer [4], [5]. Different studies showed that, risk of endometrial cancer in the setting of postmenopausal bleeding increases with increasing age after menopause [6]. Age >55 years, history of recurrent bleeding episodes, and bleeding volume exceeding five pads per day were significantly associated with endometrial cancer.

\section{RISK FACTORS FOR ENDOMETRIAL CANCER}

- Chronic oestrogen activities/ chronic anovulation.

- Unopposed oestrogen only therapy.

- Tamoxifen use - it has an anti-oestrogen effect on the breast, but a pro-oestrogen effect on the uterus and bones.

- Obesity.

- Early menarche and late menopause.

- Increasing age.
- Estrogen secreting tumour.

- Hereditary non-polyposis colorectal carcinoma.

- Lynch syndrome.

- Potential heritable factors-cowden syndrome, multiple hamartoma syndrome.

- BRCA carriers.

- Uncertain factors - diabetes, hypertension, phytoestrogen [7].

In a meta-analysis of observational studies, the pooled prevalence of postmenopausal bleeding among patients with endometrial cancer was 91 percent (95\% CI 87-93), irrespective of tumor stage [8]. Pelvic examination is usually normal in patients with early-stage disease. In more advanced disease, the uterus may be enlarged and/or fixed in the pelvis. In postmenopausal patients, uterine imaging may show a thickened endometrium and endometrial biopsy reveals diagnosis.

Polyps - Polyps are benign endometrial growths of unknown aetiology that are a common cause of perimenopausal and early postmenopausal uterine bleeding. Growth of polyps can be stimulated by estrogen therapy or tamoxifen. The definite etiology of endometrial polyps is unknown. However, endometrial polyps are associated with endometrial hyperplasia; therefore, unopposed estrogen is considered to be a risk factor [9]. The prevalence of malignant endometrial polyps in the symptomatic postmenopausal female is $4.47 \%$ compared with $1.51 \%$ in an asymptomatic postmenopausal female [10]. Additional risk factors for malignant endometrial polyps include age greater than 60, large-sized polyps, menopause status, symptomatic bleeding, and polycystic ovarian syndrome [11]. Chronic tamoxifen use has been associated with the development of endometrial hyperplasia, polyps and endometrial cancer. TVS shows, increased endometrial thickness greater than $4 \mathrm{~mm}$ is related to endometrial pathology, including polyps [12]. The location of the polyp, number, and diameter does not correlate with the reported symptoms [13].

SIS or hysteroscopy are good alternative. Hysteroscopic polypectomy with endometrial biopsy is gold standard. Blind tissue sampling with endometrial biopsy or D\&C is inaccurate in the diagnosis of endometrial polyps and should only be used in settings where hysteroscopic treatment is unavailable [14]. Histopathology showing polyp with atypia or recurrence of polyp carries potential for malignancy. Hysterectomy should be the preferred treatment in this case.

Endometrial hyperplasia $(E H)$ - One of the important causes of PMB is Endometrial hyperplasia. Since postmenopausal women should be estrogen deficient, endometrial hyperplasia at this time it is abnormal and requires a usual explanation. Low dose estrogen and micro vascular changes of endometrium by progesterone are associated with bleeding. MHT may cause spotting for couple of weeks but it usually improves by 6 months of time. Endogenous estrogen production from ovarian or adrenal tumors is also possible causes of hyperplasia. Obese women also have high levels of endogenous estrogen due to the conversion of androstenedione to estrone and the aromatization of androgens to estradiol, both of which occur in peripheral adipose tissue. Also, EH frequently results from chronic estrogen stimulation unopposed by the 
counterbalancing effects of progesterone [15].

The presence of nuclear atypia is the most important indicator of the risk of endometrial carcinoma. It is sometimes difficult to distinguish a precursor lesion from endometrial carcinoma. Endometrial hyperplasia also known as endometrial intraepithelial neoplasia.

Endometrial hyperplasia/intraepithelial neoplasia typically presents with abnormal uterine bleeding in postmenopausal women. Endometrial hyperplasia/intraepithelial neoplasm is a histologic diagnosis made with sampling of the endometrium. Neither endometrial biopsy nor dilatation and curettage detect all cases of endometrial carcinoma; up to 10 percent are falsely negative.

Leiomyomata uteri - Leiomyomata uteri (fibroids) are the most common pelvic tumours in women. The prevalence in postmenopausal women is one-tenth that of premenopausal women, thus they are a potential, but uncommon, cause of uterine bleeding in menopausal women [16]. The diagnosis of a uterine sarcoma should be considered in postmenopausal women with presumed uterine leiomyomas producing symptoms.

Sarcomas of the uterus constitute only 3 to 5 percent of all uterine tumours and may present with postmenopausal bleeding. These cancers arise from the stroma of the endometrium (endometrial stromal sarcomas) or the myometrium. They may look and feel like benign leiomyomas. Endometrial histology may be normal; diagnosis requires a hysterectomy.

Fallopian tube or ovarian cancer can cause postmenopausal uterine bleeding. Cervical and vaginal cancers typically present with vaginal bleeding. Vulvar cancers are not associated with bleeding until they are advanced.

Choriocarcinoma is a rare cause of uterine bleeding in menopausal women.

Post radiation therapy — Vaginal bleeding can be a late effect of radiation therapy [17].

Anticoagulant therapy - Use of anticoagulants may rarely cause uterine bleeding.

Herbal and dietary supplements - Soy and other phytoestrogens in large doses may be associated with $\mathrm{EH}$ [18].

Infection - Endometritis is an uncommon cause of postmenopausal bleeding. In the developing world, however, endometrial tuberculosis may present as postmenopausal bleeding [19]-[21].

Postmenopausal hormone therapy - Many postmenopausal women who take estrogen therapy develop vaginal bleeding; the frequency depends upon the regimen and the dose used. Usually within 6 months' time it gets well.

Diseases of the urethra (e.g., urethritis), bladder (e.g., cancer or urinary tract infection), and bowel (eg, inflammatory bowel disease or hemorrhoids) may cause bleeding that is mistaken for genital tract bleeding. These disorders should be considered and evaluated for in patients with bleeding in whom there is no obvious genital tract etiology. A radiograph of the pelvis to rule out fracture should be considered when there is genital bleeding after trauma, in a postmenopausal woman with osteoporosis.

\section{Evaluation}

Primary evaluation of women with postmenopausal bleeding should include detailed medical history, clinical examination, and transvaginal ultrasound (TVS) with evaluation of endometrial thickness (ET), with or without endometrial smpling [22]. According to ACOG the clinical approach to postmenopausal bleeding requires prompt and efficient evaluation to exclude or diagnose carcinoma. Women with postmenopausal bleeding may be assessed initially with either endometrial biopsy or TVS. This initial evaluation does not require performance of both tests. TVS can be useful in the triage of patients in whom endometrial sampling was performed but tissue was insufficient for diagnosis. When TVS is performed for patients with postmenopausal bleeding and an endometrial thickness of less than or equal to $4 \mathrm{~mm}$ is found, endometrial sampling is not required. Meaningful assessment of the endometrium by ultrasonography is not possible in all patients. In such cases, alternative assessment should be completed. When bleeding persists despite negative initial evaluations, additional assessment usually is indicated [23].

Endometrial cancer can reasonably be excluded by ultrasound in postmenopausal women with a thin $(\leq 4 \mathrm{~mm})$, homogeneous endometrium. Endometrial biopsy is required:

- If repeated bleeding episodes for women who showed normal findings on primary evaluation.

- The endometrial lining is thicker than $4 \mathrm{~mm}$.

- The endometrium shows diffuse or focal increased echogenicity (heterogeneity).

- The endometrium is not adequately visualized on sonography.

- The woman has persistent bleeding.

The risk of endometrial cancer is low when the endometrial thickness is less than $4 \mathrm{~mm}$ [24]-[27]. With non-representative/uncertain findings on endometrial biopsy, the cause of bleeding can be atrophic endometrial lining, however, malignancy should be excluded. Differential diagnosis of bleeding from the urinary tract or bowel should be excluded.

\section{A. History}

A detailed history on duration, frequency, length \& quantity of bleeding and its relationship to coitus, pain should be established. Menstrual history, parity, past medical history and family history are important in evaluation of PMB. Any risk factors need to be elicited. Is there a family history of breast, colon, and endometrial cancer? What is the medical history and are any medications being taken (e.g., hormones therapy, anticoagulants)?

Women with a family history of hereditary non-polyposis colorectal cancer have a lifetime risk of developing endometrial cancer of around 42-60\%. They usually develop cancer before menopause. $\mathrm{H} / \mathrm{O}$ liver diseases need to be focused.

Evaluation needs General examination and thorough physical examination of the external and internal anatomy of the female genital tract. The focus of the evaluation is to determine the bleeding site; to note any suspicious lesions, lacerations, or foreign bodies; and to assess the size, contour, and tenderness of the uterus. Lower genital tract (vulva, vagina, ectocervix) causes of bleeding can usually be 
excluded by a normal physical examination.

\section{B. Clinical examination}

- Speculum Examination

- Bi manual vaginal Examination

- Digital Rectal Examination (DRE)

\section{Investigation}

TVS to measure ET is the mainstay to evaluate endometrium. Color

Doppler is good adjustment to ET, if ET is $<4 \mathrm{~m}$ then may not require any further investigation unless there is persistent or recurrence of bleeding. If ET is $>4 \mathrm{~mm}$ endometrial biopsy must be performed. Clinical judgment should be used when there is recurrent PMB.

Definite diagnosis of PMB is made by histopathology by Endometrial sampling or D \& C. Hysteroscopy (gold standard for evaluation) adjunct investigations are done to exclude other different diseases.

Transvaginal ultrasound (TVS) is appropriate for initial evaluation of postmenopausal bleeding. If the endometrium has a thickness $\leq 4 \mathrm{~mm}$, further evaluation might not be necessary. If bleeding persists or recurs, endometrial sampling is indicated. TVS, SIS (saline infusion sonography), repeated endometrial biopsy, diagnostic hysteroscopy with endometrial biopsy can be done. Hysteroscopic evaluation and guided biopsy is the gold standard. Diagnostic hysteroscopy provides direct visualization of the endometrial cavity, thereby allowing targeted biopsy or excision of lesions identified during the procedure. A D\&C alone (approximately 60 percent of curettage specimens sample less than half of the uterine cavity so there is chance of missing pathology [28].

Obesity, uterine position, or myometrium abnormalities may preclude satisfactory endometrial assessment with TVS. In these cases, postmenopausal bleeding should be evaluated with sonohysterography, office hysteroscopy, or endometrial sampling. If such sampling yields insufficient tissue for diagnosis, further evaluation might not be necessary, provided subsequent TVS demonstrates an endometrial thickness $\leq 4 \mathrm{~mm}$ and bleeding does not recur. When postmenopausal women without bleeding undergo TVS for indications such as pelvic pain or adnexal pathology, an endometrial thickness $>4 \mathrm{~mm}$ may be incidentally found, additional endometrial assessment is not routinely indicated. Endometrial cancer can reasonably be excluded by ultrasound in postmenopausal women with a thin $(\leq 4 \mathrm{~mm})$.

Persistent bleeding can be a sign of endometrial cancer even when the endometrial thickness is less than 4 to $5 \mathrm{~mm}$ since a thin or indistinct endometrial stripe does not reliably exclude type 2 endometrial cancer [29], [30]. Therefore, women with persistent bleeding should be evaluated further.

Cervical cytology - The mean age of cervical cancer diagnosis is 52.2 years; the distribution of cases is bimodal, with peaks at 35 to 39 years and 60 to 64 years [31]. All women need cervical cancer screening as part of the evaluation of abnormal bleeding, as it can be difficult to distinguish between endocervical and upper uterine bleeding. Thin Prep, the same specimen can be used for analysis of both cytology and HPV.
Any visible lesion needs to be biopsied, even if the cytology is normal [32].

SIS, MRI, CT Scan is reserved for special cases.

\section{TREATMENT}

In postmenopausal women, uterine bleeding is usually light and self-limited. Treatment is focused on the cause. But exclusion of cancer is the main objective; However, treatment is otherwise dependent on findings during the diagnostic evaluation.

Regarding management of EH it is determined by clinical factors and by histological classification. The three most common options for the management of $\mathrm{EH}$ are surveillance, progestin therapy, and hysterectomy.

Clinical factors need to be considered are-obesity, ovulatory dysfunction, increased genetic risk, increased age. Although, EH and endometrial cancer can be observed in younger women commonly in those with polycystic ovary syndrome and body mass index $>30 \mathrm{~kg} / \mathrm{m}^{2}$ [33].

The endometrial thickness on transvaginal ultrasound $\geq 20 \mathrm{~mm}$ has a greater risk for concomitant endometrial cancer [34], [35].

We need to follow 2014 World Health Organization classification criteria. i.e.:

1. Hyperplasia without atypia.

2. Hyperplasia with atypia [36].

For postmenopausal women with EH without atypia, repeat endometrial biopsy is performed every three to six months for up to one year until the biopsy shows normal endometrium. They may be treated with the levonorgestrel releasing intrauterine system (LNG-IUS) and have rates of regression to normal endometrium of approximately 90 percent. A systematic review of 24 observational studies including 1001 participants found that treatment with LNG 52 compared with oral progestins had a higher regression rate for complex EH (92 versus 66 percent) [37]. Patients with $\mathrm{EH}$ without atypia treated with the LNG 52 have rates of regression to normal endometrium of approximately 90 percent [38].

Hysterectomy is advised if normal endometrium is not achieved, if atypical EH or endometrial carcinoma develops, or if no improvement.

Postmenopausal women having EH with atypia (endometrial intraepithelial neoplasia [EIN]) needs special counselling, as malignant potential is very high. Coexistent endometrial carcinoma may be present at hysterectomy in approximately 37 percent of women with a preoperative diagnosis of atypical EH [39]. The risk of progression of atypical EH to endometrial carcinoma is 15 to 28 percent, based on studies with up to 20 years of follow-up [40].

Hysterectomy is the choice of option, though progestin therapy with the (LNG-IUS), $52 \mathrm{mg}$ with a release rate of 20 $\mathrm{mcg}$ /day over five years, has been shown regression of atypical $\mathrm{EH}$ and grade 1 endometrial carcinoma in up to 75 to 85 percent of women [41]. However, hysterectomy is curative and thus, is still the preferred treatment.

\section{A. Maintenance Therapy}

For women with EH on progestin therapy, after regression to normal endometrium, maintenance progestin 
therapy may be required indefinitely if patients have risk factors for endometrial carcinoma (e.g., obesity, diabetes, polycystic ovary syndrome). Some women may have persistent or recurrent abnormal uterine bleeding, despite no disease progression on serial endometrial sampling. In such cases, hysterectomy may be offered.

Risk of relapse - A cohort study with long-term followup showed a relapse rate of 12.7 percent for hyperplasia without atypia [42].

A review of patients with atypical $\mathrm{EH}$ managed with progestin therapy showed a recurrence rate of 23.2 percent [41].

For patients who have a relapse of $\mathrm{EH}$, consideration is given to either continuous progestin therapy or to hysterectomy.

\section{B. Specific treatment in $P M B$}

Atrophic Vaginitis: Local oestrogen treatment is given in women suffering from vaginal atrophy [43].

Endometrial polyps: Polypectomy at hysteroscopy and histopathological examination. Simultaneous endometrium sampling for histopathology is necessary as the possibility of hyperplasia is high (e.g., $3 \%$ of endometrial polyps).

Other polyps: Polypectomy at hysteroscopy and histopathological examination.

Endometrial thickness (ET) $<4 \mathrm{~mm}$ : Follow up with a repeat ultrasonography unless continuous bleeding occurs.

ET>4 mm: Endometrial sampling with hysteroscopy. Endometrial hyperplasia without atypia responds well to cyclic progestin treatment. After 3 months a repeat endometrial biopsy may be considered to confirm absence of hyperplasia. Levonorgestrel intra-uterine system is preferred over systemic treatment. Refractory patients may need hysterectomy.

Hyperplasia with atypia - Definitive treatment for postmenopausal women is surgery, medical treatment only after in-depth counseling.

Endometrial carcinoma \& cervical carcinoma: May need surgery and/or chemotherapy or radiation therapy according to the protocol.

\section{Endometrial Safety, Bleeding \& MHT (Menopausal Hormonal Therapy)}

Adequate endometrial protection was demonstrated in MHT users with sequential and continuous micronized progesterone (MPA). The levonorgestrel-releasing intrauterine system (LNS-IUS) with estrogen has been reported to be more effective than sequential MPA/other progestins for endometrial protection.

More recently, a regimen combining the SERM, bazedoxifene, with CEE has been introduced as a progestogen-free alternative for MHT in women with a uterus. Tibolone is also used extensively as a form of MHT. It should not be used before 1 year of menopause as during this period irregular bleeding may occur.

\section{Key MesSAGES}

$>$ Postmenopausal bleeding is 'endometrial cancer until proven otherwise', although only $1-14 \%$ of such patients will actually have cancer.
$>$ Prompt evaluation is essential to find out the genital, non-gynecological and other sources. Risk factors for endometrial cancer need to be assessed for each case.

$>$ Initial evaluation is by TVS, if ET is $<4 \mathrm{~mm}$ no further evaluation is required but follow up consultation must. If ET is $>4 \mathrm{~mm}$, hysteroscopy evaluation and endometrial sampling is recommended (Gold Standard).

$>$ Blind endometrial sampling is not accurate as only reveals when endometrial cancer exceeds more than $50 \%$ of the endometrial surface area so may be done if hysteroscopy evaluation is not possible.

$>$ Higher dose of progesterone may be required for endometrial protection when higher doses of estradiol are used, for hormone therapy or in women with high BMI.

$>$ Unopposed estrogen therapy should not be use if women have uterus as it is associated with a duration and doserelated increase in risk of endometrial hyperplasia and cancer.

$>$ Endometrial protection requires an adequate dose and duration of progestogen.

$>$ Adequate doses of micronized progesterone appear to be $200 \mathrm{mg}$ per day for 10-14 days in sequential therapy and $100 \mathrm{mg}$ per day for continuous combined therapy where the estradiol dose is $2 \mathrm{mg} / 50 \mathrm{mg}$ or less.

$>$ Finally, Patient counseling and shard decision is the cornerstone of success to address postmenopausal bleeding.

\section{REFERENCES}

[1] Moodley M, Roberts C. Clinical pathway for the evaluation of postmenopausal bleeding with an emphasis on endometrial cancer detection. J Obstet Gynaecol 2004; 24:736.

[2] Prendergast EN, Misch E, Chou YA, et al. Insufficient endometrial biopsy results in women with abnormal uterine bleeding. Obstet Gynecol 2014; 123 Suppl 1:180S.

[3] Ferenczy A. Pathophysiology of endometrial bleeding. Maturitas 2003; 45:1.

[4] Van Hanegem N, Breijer MC, Khan KS, et al. Diagnostic evaluation of the endometrium in postmenopausal bleeding: an evidence-based approach. Maturitas 2011; 68:155.

[5] Cote ML, Ruterbusch JJ, Ahmed Q, et al. Endometrial cancer in morbidly obese women: do racial disparities affect surgical or survival outcomes? Gynecol Oncol 2014; 133:38.

[6] Salman MC, Bozdag G, Dogan S, Yuce K. Role of postmenopausal bleeding pattern and women's age in the prediction of endometrial cancer. Aust N Z J Obstet Gynaecol 2013; 53:484.

[7] Lee-may Chen, MD Jonathan S Berek, MD, MMS; Endometrial carcinoma: Epidemiology, risk factors, and prevention: UpTodate Nov 9, 2020.

[8] Clarke MA, Long BJ, Del Mar Morillo A, et al. Association of Endometrial Cancer Risk with Postmenopausal Bleeding in Women: A Systematic Review and Meta-analysis. JAMA Intern Med 2018; 178:1210.

[9] Tanos V, Berry KE, Seikkula J, Abi Raad E, Stavroulis A, Sleiman Z, Campo R, Gordts S. The management of polyps in female reproductive organs. Int J Surg. 2017 Jul; 43:7-16.

[10] Lee SC, Kaunitz AM, Sanchez-Ramos L, Rhatigan RM. The oncogenic potential of endometrial polyps: a systematic review and meta-analysis. Obstet Gynecol. 2010 Nov;116(5):1197-205. [PubMed].

[11] Elfayomy AK, Soliman BS. Risk Factors Associated with the Malignant Changes of Symptomatic and Asymptomatic Endometrial Polyps in Premenopausal Women. J Obstet Gynaecol India. 2015 May;65(3):186-92.

[12] Maia H, Barbosa IC, Marques D, Calmon LC, Ladipo OA, Coutinho EM. Hysteroscopy and transvaginal sonography in menopausal women receiving hormone replacement therapy. J Am Assoc Gynecol Laparosc. 1996 Nov;4(1):13-8. 
[13] Hassa H, Tekin B, Senses T, Kaya M, Karatas A. Are the site, diameter, and number of endometrial polyps related with symptomatology? Am J Obstet Gynecol. 2006 Mar;194(3):718-21.

[14] American Association of Gynecologic Laparoscopists. AAGL practice report: practice guidelines for the diagnosis and management of endometrial polyps. J Minim Invasive Gynecol. 2012 JanFeb;19(1):3-10.

[15] Hedrick Ellenson L, Ronnett BM, Kurman RJ. Precursor Lesions of Endometrial Carcinoma. In: Blaustein's Pathology of the Female Genital Tract, 6th ed, Kurman RJ, Hedrick Ellenson L, Ronnett, BM (Eds), Springer, New York 201.

[16] Paramsothy P, Harlow SD, Greendale GA, et al. Bleeding patterns during the menopausal transition in the multi-ethnic Study of Women's Health Across the Nation (SWAN): a prospective cohort study. BJOG 2014; 121:1564.

[17] Shoff SM, Newcomb PA. Diabetes, body size, and risk of endometrial cancer. Am J Epidemiol 1998; 148:234.

[18] Van Hunsel FP, Kampschöer P. [Postmenopausal bleeding and dietary supplements: a possible causal relationship with hop- and soycontaining preparations]. Ned Tijdschr Geneeskd 2012; 156: A5095.

[19] Sabadell J, Castellví J, Baró F. Tuberculous endometritis presenting as postmenopausal bleeding. Int J Gynaecol Obstet 2007; 96:203.

[20] Mengistu Z, Engh V, Melby KK, et al. Postmenopausal vaginal bleeding caused by endometrial tuberculosis. Acta Obstet Gynecol Scand 2007; 86:631.

[21] Güngördük K, Ulker V, Sahbaz A, et al. Postmenopausal tuberculosis endometritis. Infect Dis Obstet Gynecol 2007; 2007:27028.

[22] Van Hanegem N, Breijer MC, Khan KS, et al. Diagnostic evaluation of the endometrium in postmenopausal bleeding: an evidence-based approach. Maturitas 2011; 68:155-64.

[23] American College of Obstetricians and Gynecologists. ACOG Committee Opinion No. 440: The Role of Transvaginal Ultrasonography in the Evaluation of Postmenopausal Bleeding. Obstet Gynecol 2009; 114:4.

[24] Goldstein SR, Nachtigall M, Snyder JR, et al. Endometrial assessment by vaginal ultrasonography before endmetrial sampling in patients with postmenopausal bleeding. Am J Obstet Gynecol 1990; 163:11923.

[25] Varner RE, Sparks JM, Cameron CD, et al. Transvaginal sonography of the endometrium in postmenopausal women. Obstet Gynecol 1991; 78:195-9.

[26] Granberg S, Wikland M, Karlsson B, et al. Endometrial thickness as measured by endovaginal ultrasonography for identifying endometrial abnormality. Am J Obstet Gynecol 1991; 164:47-52.

[27] Gull B, Karlsson B, Milsom I et al. Can ultrasound replace diltation and curettage? A longitudinal evaluation of postmenopausal bleeding and transvaginal sonographic measurement of the endometrium as predictors of endometrial cancer. Am J Obstet Gynecol 2003; 188:401.

[28] Epstein E, Ramirez A, Skoog L, Valentin L. Dilatation and curettage fails to detect most focal lesions in the uterine cavity in women with postmenopausal bleeding. Acta Obstet Gynecol Scand 2001; 80:1131.

[29] Wang J, Wieslander C, Hansen G, et al. Thin endometrial echo complex on ultrasound does not reliably exclude type 2 endometrial cancers. Gynecol Oncol 2006; 101:120.

[30] Chandavarkar U, Kuperman J, Muderspach L, et al. Postmenopausal endometrial cancer: Reevaluating the role of endometrial echo complex. Gynecol Oncol 2011; 120:S11.

[31] http://seer.cancer.gov/csr/1975_2007/browse_csr.php?section=5\&pag e=sect_05_table.07.html (Accessed on March 14, 2011).

[32] Partridge EE, Abu-Rustum NR, Campos SM, et al. Cervical cancer screening. J Natl Compr Canc Netw 2010; 8:1358.

[33] Rosen MW, Tasset J, Kobernik EK, et al. Risk Factors for Endometrial Cancer or Hyperplasia in Adolescents and Women 25 Years Old or Younger. J Pediatr Adolesc Gynecol 2019; 32:546.

[34] Vetter MH, Smith B, Benedict J, et al. Preoperative predictors of endometrial cancer at time of hysterectomy for endometrial intraepithelial neoplasia or complex atypical hyperplasia. Am J Obstet Gynecol 2020; 222:60. e1.

[35] Karlsson B, Granberg S, Wikland M, et al. Transvaginal ultrasonography of the endometrium in women with postmenopausal bleeding--a Nordic multicenter study. Am J Obstet Gynecol 1995; $172: 1488$.

[36] Emons G, Beckmann MW, Schmidt D, et al. New WHO Classification of Endometrial Hyperplasias. Geburtshilfe Frauenheilkd 2015; 75:135.

[37] Gallos ID, Shehmar M, Thangaratinam S, et al. Oral progestogens vs levonorgestrel-releasing intrauterine system for endometrial hyperplasia: a systematic review and metaanalysis. Am J Obstet Gynecol 2010; 203:547. e1.
[38] Abu Hashim H, Ghayaty E, El Rakhawy M. Levonorgestrel-releasing intrauterine system vs oral progestins for non-atypical endometrial hyperplasia: a systematic review and metaanalysis of randomized trials. Am J Obstet Gynecol 2015; 213:469.

[39] Rakha E, Wong SC, Soomro I, et al. Clinical outcome of atypical endometrial hyperplasia diagnosed on an endometrial biopsy: institutional experience and review of literature. Am J Surg Pathol 2012; 36:1683.

[40] Reed SD, Newton KM, Garcia RL, et al. Complex hyperplasia with and without atypia: clinical outcomes and implications of progestin therapy. Obstet Gynecol 2010; 116:365.

[41] Gunderson CC, Fader AN, Carson KA, Bristow RE. Oncologic and reproductive outcomes with progestin therapy in women with endometrial hyperplasia and grade 1 adenocarcinoma: a systematic review. Gynecol Oncol 2012; 125:477.

[42] Gallos ID, Krishan P, Shehmar M, et al. Relapse of endometrial hyperplasia after conservative treatment: a cohort study with longterm follow-up. Hum Reprod 2013; 28:1231.

[43] Suckling JA, Kennedy R, Lethaby A, et al. Local oestrogen for vaginal atrophy in postmenopausal women. Cochrane Database of Syst Rev 2006, (4).

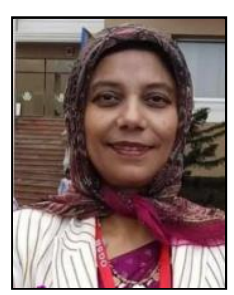

Prof. Shaikh Zinnat Ara Nasreen was born in Dhaka, Bangladesh. She passed MBBS from Dhaka Medical College \& Hospital in 1984 and got gold medal for record marks. Later she achieved FCPS, MPH, MRCOG \& FRCOG.

She is now working as Professor \& Head of the Department of Obs. \& Gyne, Z. H. Sikder Womens' Medical College \& Hospital, West Dhanmondi, Dhaka, Bangladesh.

Prof. Nasreen has many national and international

publications.

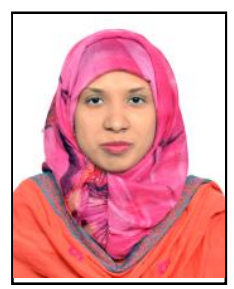

Dr. Nusrat Mahjabeen was born in Dhaka, Bangladesh. She passed MBBS from Ibrahim Medical College \& BIRDEM Hospital in 2007. She acquired MS (Masters in surgery) in Obs. \& Gynae under Dhaka University in 2017.

She is now working as Assistant Professor in the Department of Obs. \& Gyne, Z. H. Sikder Womens' Medical College \& Hospital, West Dhanmondi, Dhaka, Bangladesh.

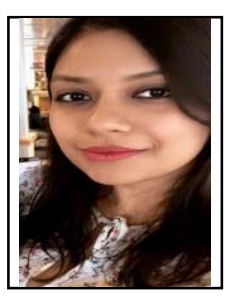

Dr. Safana Shahreen was born in Dhaka Bangladesh. She passed MBBS from AFMCH and got chancellor award (gold medal) for record marks. She is now working as Registrar in the Department of Luton Hospital, UK. 